\title{
TRANSVERSE WAKEFIELDS FROM TAPERED COLLIMATORS: MEASUREMENTS AND ANALYSIS
}

\author{
P. Tenenbaum, K. Bane, L. Eriksson, R.K. Jobe, D. McCormick, C.K. Ng, T.O. Raubenheimer, \\ M.C. Ross, G. Stupakov, D. Walz, SLAC * \\ D. Onoprienko, Brunel University, UK
}

\begin{abstract}
We report on a series of measurements of the transverse wakefield from tapered collimators. The collimators were designed to principally present a geometric impedance to the beam, and to minimize impedances from resistivity or surface features; in addition, the geometries of the collimators were selected to permit examination of the scaling behavior of the wakefield due to collimator taper angle and minimum gap size. We present the measured near-center wakefields of the collimators, as well as the effect of bunchlength variation. The measurements are compared to analytic models and MAFIA simulations.
\end{abstract}

\section{INTRODUCTION}

In contemporary high-performance accelerators, collimators are commonly used to remove large-amplitude particles from the beam and to protect downstream components from bunches which are following an unsafe trajectory; it is anticipated that future accelerators will continue to require mechanical collimators for these purposes. In order to accomplish machine protection or halo removal, the collimators typically must be positioned quite close to the beam; this in turn implies that the collimators can present a large impedance, which can destroy the high-quality beams required for applications such as $\mathrm{x}$-ray lasers and linear colliders.

In principle, the geometric impedance presented by a beam collimator can be reduced by introducing a longitudinal taper. In practice, it is difficult to either simulate or calculate the high-frequency impedance of a large, tapered collimator with sufficient accuracy to satisfactorily model its impact on beam dynamics, and experimental measurements of such objects are also scarce.

In order to address the absence of experimental data, we have designed, constructed, and installed a dedicated beam test chamber for collimators at the $1.19 \mathrm{GeV}$ point in the SLAC linac [1]. This chamber has been used to measure the transverse wakefields of a series of tapered collimators. We report on the results of these measurements, and also compare them to recent analytic and simulation-based predictions.

\footnotetext{
* Work Supported by the Department of Energy, Contract DE-AC03-
} $76 \mathrm{SF} 00515$.

\section{APPARATUS AND MEASUREMENT TECHNIQUE}

The test chamber is a rectangular vacuum chamber which can hold up to 4 test collimators at one time. During measurements, one of the collimators is positioned in the path of the beam by a remote-controlled translation stage. Once a collimator has been positioned in the beam's path, the wakefield is measured by varying the collimator's vertical position and measuring the resulting vertical deflection of the beam centroid on downstream beam position monitors (BPMs). The vertical position is varied using a remote-controlled high-precision translation stage built for the Final Focus Test Beam (FFTB) project at SLAC; it has a range of motion in the vertical of $\pm 1.4 \mathrm{~mm}$, and a positioning resolution of $1 \mu \mathrm{m}$ [2].

\section{COLLIMATOR APERTURES}

The first set of collimators was designed to test the geometric wakefield of a tapered aperture. In order to achive an accurate measurement of the desired effect, it was necessary to minimize the impedance of each collimator from other sources - notably the surface roughness and finite conductivity of the apertures. This was accomplished by constructing each aperture from oxygen-free, elemental copper, and carefully machining each piece to achieve an adequately smooth finish.

Each of the four collimators has a maximum size of 38 $\mathrm{mm}$ high by $38 \mathrm{~mm}$ wide. Three of the collimators were constructed with a rectangular aperture at the minimum, and these collimators are tapered in the vertical but not the horizontal. One of the collimators is tapered in the vertical and the horizontal to achieve a square aperture: this collimator is an approximation of a tapered round collimator, the transverse wakefield for which has been predicted by Yokoya [3]. Table 1 indicates the geometry of the collimators, parameterized by taper angle $\alpha$ and minimum halfgap $b_{1}$. Note that in previous publications, the parameters of collimators (1) and (3) were exchanged: this was due to an oversight during installation $[4,5]$. Table 1 reflects the correct configuration of the apparatus.

At the time of construction, the theory used to estimate the deflections [6] indicated that the rectangular collimators would produce extremely large kicks - tens of microradians per millimeter of beam-to-collimator offset. More recently, it has become clear that the applicability of the expressions used for those predictions are quite limited, and that rectangular collimators with large width to height ratio 
Table 1: Parameters of the tapered collimators. All rectangular collimators have a half-width, $h$, of $19 \mathrm{~mm}$.

\begin{tabular}{|l|c|c|c|c|}
\hline Coll \# & 1 & 2 & 3 & 4 \\
\hline \hline Type & Rect & Square & Rect & Rect \\
\hline$b_{1}, \mathrm{~mm}$ & 1.9 & 1.9 & 1.9 & 3.8 \\
\hline$\alpha, \mathrm{mrad}$ & 168 & 335 & 335 & 298 \\
\hline
\end{tabular}

fall into three qualitatively different regimes [7]:

- For extremely shallow tapers, in which $\sqrt{\alpha h^{2} / b_{1} \sigma_{z}}<3.1$, the transverse impedance of the collimator is purely inductive and is proportional to $\alpha h / \sigma_{z} b_{1}^{2}$

- For extremely steep tapers, for which $\sqrt{\alpha b_{1} / \sigma_{z}}>$ 0.37 , the transverse impedance of the collimators is proportional to $1 / b_{1}^{2}$ (this is known as the diffractive regime)

- For tapers between the inductive and diffractive regimes, the impedance is proportional to $\sqrt{\alpha / \sigma_{z} b_{1}^{3}}$.

The diffractive model predicts an impedance which is independent of taper angle, and constitutes an upper bound on the impedance of a collimator with a given half-gap $b_{1}$. The inductive impedance model, when applied to the rectangular collimators in Table 1, predicts impedances which are much larger than those predicted by the diffractive model, which indicates that these collimators are far from the region of applicability of the inductive model. All three rectangular collimators are within the region where the diffractive model is expected to be applicable.

Like rectangular collimators, round collimators fall into inductive or diffractive regimes of transverse impedance (although they do not have an intermediate regime between these two, which arises in the rectangular collimator due to its asymmetry). In this case the inductive regime has a larger region of applicability, and the square collimator in Table 1 is believed to be modelled by the inductive impedance model. Note that all four collimators are near the transitions between different regimes; the accuracy of the analytic estimates is therefore somewhat limited.

\section{WAKEFIELD MEASUREMENTS AND ANALYSIS}

The majority of wakefield measurements were performed with electrons, at a bunch charge of approximately $2 \times 10^{10}$, and an RMS bunch length of $650 \mu \mathrm{m}$, which corresponds to full compression of the electron bunch from the SLAC damping ring. A subset of measurements was performed at lower charge (approximately $1.2 \times 10^{10}$ ), another subset was performed at a larger bunch length (approximately $1.2 \mathrm{~mm}$ RMS), and a third subset was performed with positrons.

\subsection{Measurement Procedure}

The BPMs in sector 2 of the SLAC linac typically have significant DC offsets: during normal running, the RMS of BPM values in the region is several hundred micrometers. These DC offsets must be subtracted from the measurement data in order to accurately measure the beam kicks from the collimator wakefields. The DC offsets are determined by reading out the BPMs for 100 pulses while the apparatus is held stationery at its nominal zero point. The average of the 100 pulses, called the "reference orbit," is subtracted from all subsequent data taken at different vertical positions of the collimator.

The collimator vertical position is scanned over the range of the translation stage $( \pm 1.4 \mathrm{~mm})$, typically in steps of 0.2 $\mathrm{mm}$. At each position of the collimator, the BPMs are read out for 12 to 25 pulses. On each pulse of scan data the reference orbit is subtracted and the $\mathrm{R}$ matrices in sector 2 are used to fit the incoming and outgoing position and angle of the beam at the collimator $z$ location: this is necessary to include the effect of pulse-to-pulse beam jitter in sector 2. Cuts are made on pulses which exhibit pathologies such as low bunch charge or poor quality of fit to the model $\mathrm{R}$ matrices. The remaining pulses at each collimator position are averaged together, and an average deflection angle for each position is determined. Figure 1 shows the measured deflection angle as a function of beam-to-collimator offset for collimator 3 . Note that over the range of mover positions used, the wakefield shows the nonlinear "nearwall" behavior associated with collimator wakefields [6]. Also shown in Figure 1 is a fit to an odd-power polynomial: $y=p 0+p 1 x+p 2 x^{3}$.

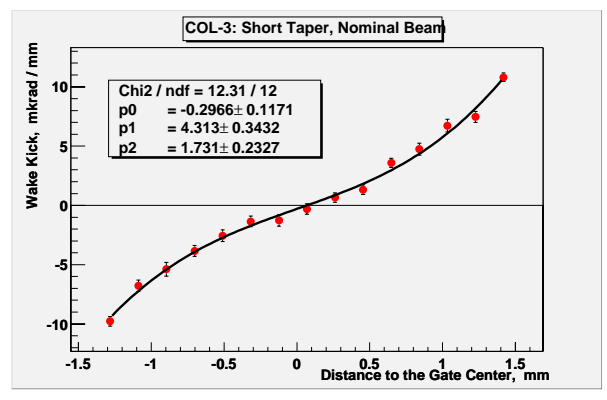

Figure 1: Deflection angle versus beam-to-collimator offset for collimator 3 .

\subsection{Measurement Results}

Table 2 shows the measured wakefield kick to the beam centroid, in $\mathrm{V} / \mathrm{pC} / \mathrm{mm}$, from each of the collimators. As in Figure 1, the results in Table 2 were obtained by performing an odd-power third-order polynomial fit to the data, and collecting the first-power coefficients; the exception to this is collimator 4, which has such a large aperture that the cubic term was negligible. Table 2 also shows the expected deflection for the standard RMS bunch length of $650 \mu \mathrm{m}$; in the case of collimator 2 , the analytic models for a round 
collimator are used. While the predictions for all collimators are comparable to the measured values, in the case of collimators 1 and 3 the actual deflection is somewhat smaller than the prediction; this is to be expected, since the diffractive model essentially treats the collimator as untapered; the tapers in collimators 1 and 3, while steep, do reduce the geometric impedance somewhat.

Table 2 also shows the measured wakefields for an RMS bunch length of $1.2 \mathrm{~mm}$. Both over- and under-compressed bunches were used for this measurement, and both sets of measurements are shown, along with analytic-model predictions.

Although additional data was taken for nominal bunch length and reduced charge, these measurements simply confirmed that the deflection scaled linearly with charge, and so we have not included them here. Additional measurements taken with positrons were in qualitative agreement with the electron data (i.e., the deflection had the correct sign for a transverse wakefield, but not for an electrostatic or magnetostatic effect); however, the beam conditions for the positron measurements were not well controlled, and so the positron measurements are also not included in Table 2. show agreement at the level of a factor of 2 with improved analytic models. The measured wakefields are smaller and less sensitive to bunch length than was originally expected; this is because the longitudinal tapers are so steep that the collimators are in the diffractive regime and not the inductive regime of transverse geometric impedance, a fact that was not apprehended at the time of construction.

The apparatus used for these studies is a general purpose test stand for the measurement of collimator wakefields. We are improving the data acquisition and analysis procedures, and expect to achieve more precise measurements. In the near future, a tapered graphite collimator made by DESY will be studied, and a more general study of resistive wakefields will follow. Other tests, including a test of an NLC prototype collimator and tests of impedance due to surface finish, are also foreseen.

\section{ACKNOWLEDGEMENTS}

The wakefield experiment could not have been performed without the efforts of SLAC's technical experts, in particular B. Brugnoletti, K. Dudley, and M. Ortega. We would also like to thank D. Burke and T. Markiewicz for their support of this experiment.

Table 2: Measured, theoretical, and simulation wakefield results for nominal and long-bunch conditions. A plus sign (“+”) in the bunch length indicates over-compression, a minus sign (“-”) under-compression.

\begin{tabular}{|l|c|c|c|c|c|}
\hline & & \multicolumn{4}{|c|}{ Collimator } \\
& $\sigma_{z}, \mathrm{~mm}$ & 1 & 2 & 3 & 4 \\
\hline \hline Meas & 0.65 & $1.2 \pm 0.1$ & $1.4 \pm 0.1$ & $1.4 \pm 0.1$ & $0.54 \pm 0.05$ \\
\hline Theo & 0.65 & 2.5 & 1.4 & 2.5 & 0.6 \\
\hline Sim & 0.65 & 1.1 & 2.1 & 1.3 & 0.4 \\
\hline \hline Meas & $1.2-$ & $0.78 \pm 0.13$ & $1.2 \pm 0.1$ & $1.08 \pm 0.09$ & $0.49 \pm 0.14$ \\
\hline Meas & $1.2+$ & $0.83 \pm 0.12$ & $1.3 \pm 0.2$ & $1.04 \pm 0.16$ & $0.44 \pm 0.10$ \\
\hline Theo & 1.2 & 2.5 & 0.75 & 2.5 & 0.6 \\
\hline Sim & 1.0 & 0.85 & & 1.1 & 0.4 \\
\hline Sim & 1.3 & & & 1.0 & \\
\hline
\end{tabular}

Table 2 also includes the wakefield predicted by simulation studies of tapered collimators. The majority of these simulations used the program MAFIA [8], and are discussed in greater detail elsewhere [9]. The simulation of collimator 2 used the program ABCI [10], and assumed a round geometry rather than a square one. The MAFIA simulation results are generally quite close to the measured wakefields; the ABCI simulation predicts a wakefield for the square collimator which is $50 \%$ larger than the measured value.

\section{CONCLUSIONS}

The wakefields of a set of longitudinally-tapered collimators have been measured in the SLAC linac, and compared to analytic and simulation models. The measured wakefields typically agree well with simulation results, and

\section{REFERENCES}

[1] P. Tenenbaum et al, Proceedings PAC-99, 3453 (1999).

[2] G.B. Bowden et al, NIM A368:579 (1996).

[3] K. Yokoya, CERN-SL/90-88 (AP) (1990).

[4] P. Tenenbaum it et al, Proceedings EPAC-2000 (2000).

[5] P. Tenenbaum it et al, Proceedings LINAC-2000, 56 (2000).

[6] G. Stupakov, SLAC-PUB-7167 (1996).

[7] G. Stupakov, these proceedings.

[8] R. Klatt et al, "MAFIA - a Three Dimensional Electromagnetic CAD System for Magnets, RF Structures, and Transient Wake Field Calculations," Proceedigs LINAC-86, 276 (1986).

[9] C.K Ng et al, these proceedings.

[10] Y.H. Chin, “Users' Guide for ABCI Version 8.7," CERNSL-94-02-AP (1994). 